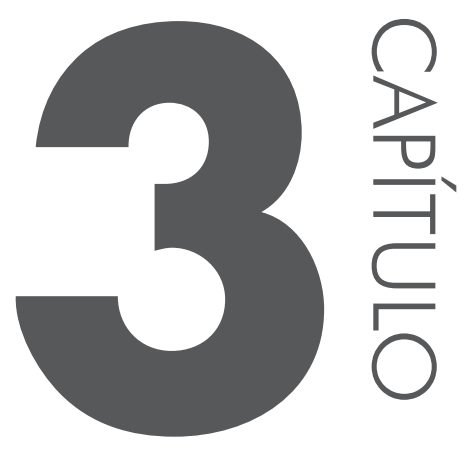

\title{
MÉTODO DOS ELEMENTOS FINITOS
}

\subsection{INTRODUC̣ÃO}

O método dos elementos finitos (MEF) é uma técnica de análise numérica que tem por objetivo obter soluções aproximadas de problemas regidos por equações diferenciais. Ele foi desenvolvido, inicialmente, para o cálculo de tensões em sistemas estruturais. Entretanto, tem sido utilizado em uma vasta gama de problemas de Transmissão de Calor e Eletromagnetismo, de Mecânica dos Fluídos e de Mecânica dos Sólidos.

O MEF possui uma difusão muito grande, seja no meio acadêmico, seja no industrial, disponibilizado em códigos comerciais como NASTRAN ${ }^{\circledR}$, ABAQUS $^{\circledR}$, SYSTUS $^{\circledast}$, ANSYS $^{\circledR}$, entre outros. Neste trabalho, foi utilizado o ANSYS $^{\circledR}$, versão 6.0.

As soluções analíticas fechadas de alguns problemas de engenharia de razoável complexidade são inviáveis ou mesmo impossíveis. A estratégia de se proceder a uma simplificação do problema de modo que se possa construir um modelo matemático de solução analítica possível é uma das alternativas para se abordar o problema, mas que pode levar, quase que invariavelmente, a imprecisões nos 
resultados. O crescente aumento da capacidade de processamento dos computadores tem possibilitado, como alternativa às soluções analíticas, a preservação da complexidade do modelo e a utilização de técnicas aproximadas de resolução, nas quais se insere o MEF.

\subsection{FUNDAMENTOS DO MEF}

Nos problemas em que a abordagem se faz pela formulação em domínios contínuos, as variáveis de campo - a exemplo das tensões, deformações etc. assumem independentemente seus valores em cada ponto de seus respectivos domínios. As soluções para esse tipo de problema em que uma função associa um valor da variável de campo para cada coordenada espacial em todos os pontos do domínio são denominadas soluções analíticas.

Transformando um problema infinito-dimensional em um problema com número finito de incógnitas, por meio de um processo de discretização, o MEF divide o domínio, no qual o problema é estudado, em elementos, conforme se pode verificar na Figura 3.1.

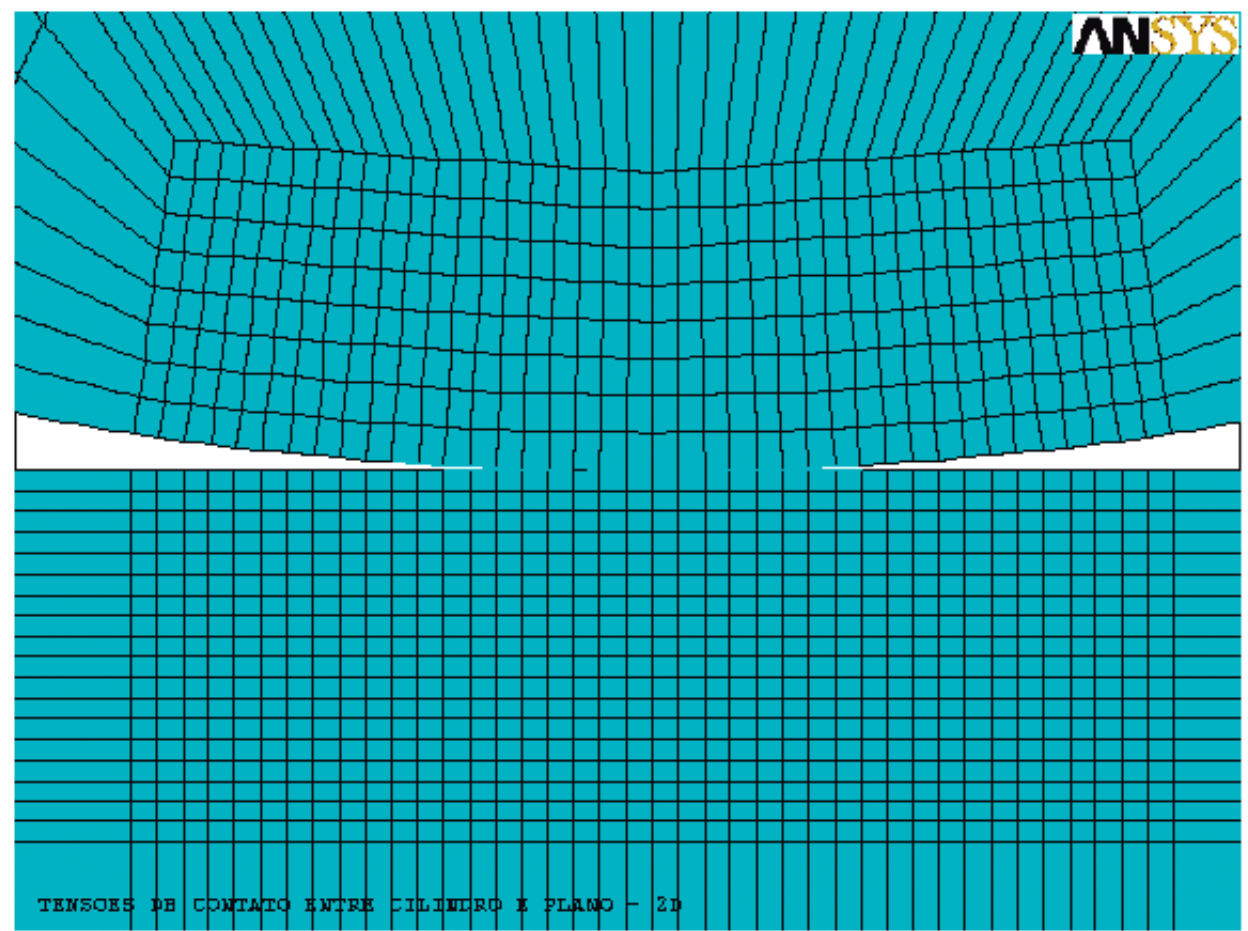

Figura 3.1 Discretização em elementos quadrilaterais em uma região de contato entre um cilindro e um plano. 
Cada elemento possui pontos em seu interior ou em sua fronteira denominados nós, em que soluções aproximadas para as variáveis de campo são expressas como funções arbitrárias dos valores assumidos pelas incógnitas, chamados valores nodais. Essas funções são denominadas funções de forma ou funções de interpolação. As incógnitas do problema passam a ser os valores das variáveis de campo nos pontos nodais, os quais compõem um conjunto finito nesse processo de discretização. Ressalta-se que a discretização leva a modelos expressos sob a forma de sistemas de equações diferenciais ordinárias no caso de problemas de propagação (dinâmica) ou de equações algébricas no caso de problemas de equilíbrio. Uma vez resolvidas numericamente tais equações, a avaliação das variáveis de campo dentro de cada elemento pode, então, ser feita pelas funções de forma, sendo que, do número de nós e das funções arbitradas, depende a precisão do modelo e, consequentemente, o esforço computacional. Na Seção 4.2.5, há maiores detalhes sobre as características pertinentes ao modelo adotado, como o número de graus de liberdade.

Ao se proceder a uma discretização de domínios em modelos que possuam geometrias complexas, a utilização de elementos de formas, tamanhos e propriedades físicas diferentes se apresenta como uma grande vantagem do MEF - principalmente onde há problemas que envolvam domínios não homogêneos, como é caso do problema de contato entre superfícies revestidas.

Outra vantagem é a possibilidade da formulação matricial, que é um facilitador na sua implementação computacional em 3 etapas subsequentes, quais sejam:

- pré-processamento, em que se procede à construção do modelo, por geração direta, pelo próprio usuário, ou por modelagem sólida (terminologia específica do $\mathrm{ANSYS}^{\circledR}$ ), quando a geração de nós e de elementos é feita automaticamente pelo programa, procedimento mais recomendado em casos de problemas mais complexos. Ele é feito mediante os seguintes procedimentos:

1. Divisão do domínio em elementos, escolhendo o tipo e o número de elementos adequados à geometria e aos aspectos físicos do problema;

2. Opção pelas funções de interpolação que descrevem as variáveis de campo, sejam escalares, vetoriais ou tensoriais. A construção das matrizes elementares, por meio da definição das relações matriciais que descrevem o comportamento de cada elemento, individualmente, diz respeito, mais especificamente, ao processo de formulação dos elementos. Para tanto, podem ser utilizados um dos três processos a seguir:

a) Processo direto, que permite uma interpretação física do significado das matrizes elementares, embora limitado na complexidade dos problemas a que pode ser aplicado; 
b) Processo dos resíduos ponderados, baseado integralmente na matemática e muito poderoso;

c) Processo variacional, baseado no cálculo variacional.

As equações elementares, para um elemento genérico $i$, são escritas para problemas de equilíbrio:

$$
\left[k_{i}\left(\left\{u_{i}\right\}\right)\right]\left\{u_{i}\right\}=\left\{q_{i}\right\}, \quad i=1,2,3, \ldots N
$$

sendo que:

- $\left[k_{i}\left(\left\{u_{i}\right\}\right)\right]$ é a matriz de rigidez elementar;

- $N$ é o número de elementos total do modelo;

- $\left\{q_{i}\right\}$ e $\left\{u_{i}\right\}$ são os vetores dos esforços e dos deslocamentos nodais, respectivamente.

Em virtude de se estar admitindo comportamento não linear, a matriz de rigidez é dependente dos deslocamentos.

Montagem do sistema global de equações, cujas etapas se compõem de:

- imposição do equilíbrio dos esforços nos nós,

- compatibilidade dos deslocamentos nodais.

As equações de equilíbrio do modelo, escritas no sistema de coordenadas globais e obtidas por essa compatibilidade de deslocamentos dos nós e da imposição dos esforços nodais, são as que seguem:

$$
[k(\{u\})]\{u\}=\{q\}
$$

sendo que $[k(\{u\})]$ é a matriz de rigidez global;

onde $\{q\}$ e $\{u\}$ são os vetores dos esforços e dos deslocamentos nodais, respectivamente, expressos em coordenadas globais, em que o número de graus de liberdade do modelo (GDL) é dado pelo número de equações que compõem a Equação (3.2).

Para se exemplificar a formulação das equações governantes do MEF e a posterior determinação das matrizes de rigidez de contato, considere-se o caso bidimensional de contato entre um corpo alvo e um contator, esquematicamente ilustrado na Figura 3.2: 


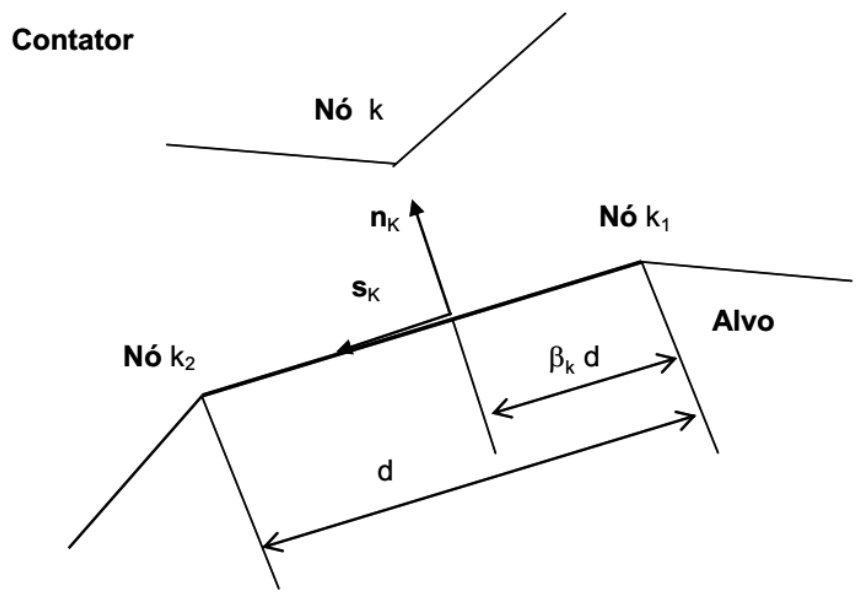

Figura 3.2 Caso bidimensional de contato, segundo Bathe (1986).

Observe que os nós $k_{1}$ e $k_{2}$ não são necessariamente as extremidades de um elemento, mas quaisquer dois nós adjacentes que pertençam ao corpo alvo do contato. A discretização das equações da mecânica do contínuo obtidas pelo princípio do trabalho virtual para $N$ corpos são dadas por:

$$
{ }^{t+\Delta t} \mathrm{~F}\left({ }^{t+\Delta t} \mathrm{U}\right)={ }^{t+\Delta t} \mathrm{R}-{ }^{t+\Delta t} \mathrm{R}_{c}\left({ }^{t+\Delta t} \mathrm{U},{ }^{t+\Delta t} \mathrm{~T}\right)
$$

e

$$
{ }^{t+\Delta t} \mathrm{~F}_{c}\left({ }^{t+\Delta t} \mathrm{U},{ }^{t+\Delta t} \mathrm{~T}\right)=0
$$

sendo que, para $m$ nós contatores,

$$
{ }^{t+\Delta t} \mathrm{~T}^{T}=\left[\lambda_{1}, \tau_{1}, \ldots, \lambda_{k}, \tau_{k}, \ldots, \lambda_{m}, \tau_{m}\right]
$$

Para o nó contator $k$ e o correspondente nó alvo, o vetor força nodal, conforme definições da Figura 3.2, é dado por:

$$
{ }^{t+\Delta t} \mathrm{R}_{k}^{c}=\left[\begin{array}{l}
-\lambda_{k}\left(\mathrm{n}_{k}+\mu \tau_{k} \mathrm{~s}_{k}\right) \\
\left(1-\beta_{k}\right) \lambda_{k}\left(\mathrm{n}_{k}+\mu \tau_{k} \mathrm{~s}_{k}\right) \\
\beta_{k} \lambda_{k}\left(\mathrm{n}_{k}+\mu \tau_{k} \mathrm{~s}_{k}\right)
\end{array}\right]
$$

Após uma linearização, no tempo $t$ obtém-se (ver maiores detalhes em BATHE, 1986):

$$
\left[\begin{array}{cc}
\left({ }^{t} \mathrm{~K}+{ }^{t} \mathrm{~K}_{u u}^{c}\right){ }^{t} \mathrm{~K}_{u \tau}^{c} \\
{ }^{t} \mathrm{~K}_{\tau u}^{c} & { }^{t} \mathrm{~K}_{\tau \tau}^{c}
\end{array}\right]\left[\begin{array}{c}
\Delta \mathrm{U} \\
\Delta \mathrm{T}
\end{array}\right]=\left[\begin{array}{c}
{ }^{t+\Delta t} \mathrm{R}-{ }^{t} \mathrm{~F}-{ }^{t} \mathrm{R}_{c} \\
-{ }^{t} \mathrm{~F}_{c}
\end{array}\right]
$$


onde as matrizes de rigidez de contato são dadas pelas seguintes expressões:

$$
\begin{array}{ll}
{ }^{t} \mathrm{~K}_{u u}^{c}=\frac{\partial^{t} \mathrm{R}_{c}}{\partial^{t} \mathrm{U}} ; & { }^{t} \mathrm{~K}_{u \tau}^{c}=\frac{\partial^{t} \mathrm{R}_{c}}{\partial^{t} \mathrm{~T}} \\
{ }^{t} \mathrm{~K}_{\tau u}^{c}=\frac{\partial^{t} \mathrm{~F}_{c}}{\partial^{t} \mathrm{U}} ; & { }^{t} \mathrm{~K}_{\tau \tau}^{c}=\frac{\partial^{t} \mathrm{~F}_{c}}{\partial^{t} \mathrm{~T}}
\end{array}
$$

A formulação da mecânica do contínuo adotada leva em conta muitas condições gerais de deformações e relações constitutivas, incluindo a lei de atrito de Coulomb. Evidentemente, essa formulação é também aplicável ao contato sem atrito. Nesse caso, as equações do MEF têm somente forças normais nos nós contatores, como se sabe.

De particular interesse na solução dos problemas de contato é a capacidade do algoritmo de convergir naqueles casos em que se têm geometrias, deformações e condições de contato complexas. Face ao exposto, vale ressaltar que passos incrementais muito grandes podem levar a grandes dificuldades de convergência nas iterações de equilíbrio, porque o estado intermediário de predição está muito distante da solução. Por outro lado, a convergência quadrática completa, quando próxima da solução, pode não ser atingida se a tangente da matriz dos coeficientes não for suficientemente suave, como resultado de uma saliência na superfície alvo, por exemplo.

- processamento, em que, estando o modelo representado por um conjunto de equações algébricas ou diferenciais, lineares ou não lineares, os valores das variáveis de campo, em cada nó, serão determinados por meio da resolução dessas equações, pelos métodos numéricos apropriados a cada caso. Nessa etapa, as condições de contorno são aplicadas ao modelo, seja pela aplicação das restrições aos deslocamentos, seja pela aplicação de deslocamentos ou das forças externas no modelo. Cálculos complementares poderão ser necessários para a obtenção de grandezas dependentes das variáveis de campo.

- pós-processamento, em que se procede a uma apresentação gráfica e/ou numérica dos resultados.

\subsection{APLICAC̣ÕES E LIMITAC̣ÕES DO MÉTODO DOS ELEMENTOS FINITOS}

São três as categorias de problemas em que se podem dividir as aplicações do MEF:

- aqueles em que se deseja calcular frequências naturais e modos de vibração de meios sólidos e fluidos, os de estabilidade linear (flambagem), bem 
como os da aeroelasticidade, estão inseridos nos denominados problemas de autovalor;

- os que tratam da caracterização do comportamento do sistema mecânico em função do tempo, em regime transitório, são denominados problemas de propagação, incluindo-se nestes a determinação das distribuições de temperaturas geradas por uma fonte de calor variável;

- os de equilíbrio, os quais têm soluções independentes do tempo. Nesta classe de problemas, inserem-se os da Mecânica dos Fluidos, que tratam da distribuição de pressão e velocidades em regime permanente, bem como os da Mecânica dos Sólidos que se preocupam com a determinação de tensões e deformações em elementos estruturais, como no caso da Mecânica do Contato.

Embora seja o MEF uma poderosa ferramenta de utilização nos problemas de Engenharia, não se deve perder de vista que é um método aproximado, o que faz com que traga em seu contexto simplificações de um determinado modelo físico que poderão acarretar resultados incoerentes com a realidade. Portanto, um equilíbrio entre as simplificações no modelo de elementos finitos - que podem levar a imprecisões nos resultados - e o excesso de rigor nas suas complexidades que podem acarretar um custo computacional inviável - é a forma mais segura de obtenção do êxito na aplicação desse método. Por esse motivo, o conhecimento das prováveis fontes de incerteza inerentes à modelagem do MEF, tais como linearidades, imperfeições na representação geométrica dos domínios mais complexos, erros de natureza numérica, entre outros, bem como o domínio do problema físico em estudo e do próprio método pelo engenheiro, são de fundamental importância na validação e na interpretação dos resultados obtidos. 
